\title{
Prognostic factors for TB-associated uveitis in the Asia-Pacific Region: results of a modified Delphi survey
}

\author{
Soumyava Basu (i) ${ }^{1} \cdot$ Rina La Distia Nora ${ }^{2} \cdot$ Narsing A Rao ${ }^{3} \cdot$ Xuejuan Jiang ${ }^{4}$ \\ Ahmad Fuady ${ }^{5}$ the International Ocular TB Study Group
}

Received: 9 July 2019 / Revised: 16 October 2019 / Accepted: 4 November 2019 / Published online: 2 January 2020

(c) The Author(s), under exclusive licence to The Royal College of Ophthalmologists 2020

\begin{abstract}
Background Prognostic factors for TB-associated uveitis (TBU) remain mostly unknown, due to the challenges in interpretation of patient data. We present consensus list of prognostic factors for resolution of inflammation in TBU, by experts across the Asia-Pacific region.

Methods We applied a modified Delphi technique to generate consensus on prognostic factors influencing the resolution of inflammation in TB-associated: anterior uveitis (AU), retinal vasculitis (RV), and multifocal serpiginoid choroiditis (MSC). The initial questionnaire was developed through a face-to-face meeting. Sixteen uveitis experts from eleven Asia-Pacific countries were included. A single investigator circulated the questionnaire electronically and received the responses. Participants scored each item on 4-point Likert scale, in three successive rounds. After each round, a number of items were reduced based on response, and summary of responses was provided to participants. At the end of Round 3, items were considered significant if they: (1) achieved a median $\geq 2$, and interquartile range $\leq 1$, and (2) $\geq 75 \%$ of the respondents agreed on whether the item was a positive or negative prognostic factor.

Results Forty-two putative questionnaire items were considered. At the end of Rounds 3, 4, 9, and 8 items in AU, RV, and MSC, respectively, met significance criteria. These included duration of disease, previous corticosteroid/immunosuppressive therapy, co-existent HIV, disease-specific imaging features, multidrug resistant TB, and duration of anti-TB therapy.

Conclusions Consensus were achieved on multiple ocular and systemic factors that influence resolution of inflammation in TBU. These will form the groundwork for validation in prospective clinical trials.
\end{abstract}

Members of the International Ocular TB Study Group are listed below Author Contributions

Supplementary information The online version of this article (https:// doi.org/10.1038/s41433-019-0743-1) contains supplementary material, which is available to authorized users.

$\triangle$ Soumyava Basu

eyetalk@gmail.com

1 Mithu Tulsi Chanrai Campus, L V Prasad Eye Institute, Bhubaneswar, India

2 Department of Ophthalmology, Universita Indonesia \& Cipto Mangunkusumo Hospital Kirana, Jakarta, Indonesia

3 Department of Ophthalmology, Keck School of Medicine, University of Southern California, Los Angeles, CA, USA

4 Department of Ophthalmology and Preventive Medicine, Keck School of Medicine, University of Southern California, Los Angeles, CA, USA

5 Department of Community Medicine, Faculty of Medicine, Universitas Indonesia, Jakarta, Indonesia

\section{Introduction}

Tuberculosis (TB) is a common cause of intraocular inflammation in several countries of the Asia-Pacific region. This region accounts for 58\% of the global TB burden, the largest for any part of the world [1]. TB-associated uveitis (TBU) has several clinical manifestations that are generally diagnosed on the basis of specific clinical signs, ancillary tests supportive of TB and exclusion of non-TB entities [2]. During the past two decades, several studies have attempted to analyze the diagnostic criteria and treatment paradigms for intraocular TB, both in TB-endemic and nonendemic countries [3-11]. However, no attempt has been made to specifically evaluate prognostic factors for this condition.

Determining prognostic factors for TBU from analyses of patient data remains challenging, as the end points of treatment are not clear. Previous studies have shown that patients can have recurrent intraocular inflammation, despite prolonged treatment with anti-TB therapy [5-12]. 
Conversely, patients diagnosed with TBU based on current diagnostic criteria, may occasionally have complete resolution with corticosteroid therapy only [5]. Such anomalous responses could be attributed to an intraocular autoimmune T-cell response that co-exists with the anti-mycobacterial immune response in the eye [13]. Thus, if the predominant immune response is autoimmune, it may resolve with corticosteroid therapy or recur in the absence of prolonged antiinflammatory therapy. In addition to the above, resolution of intraocular inflammation in TBU does not always correlate with improved visual outcomes, as the visual status is determined by multiple factors, in addition to severity of the inflammation [14]. Thus, there is a need to consider alternative strategies for determining prognostic factors during management of intraocular TB.

To resolve this dilemma, we applied the consensus-based Delphi technique for investigating prognostic factors for intraocular TB. The Delphi technique aims at deriving quantitative estimates from qualitative approaches such as the extent to which experts agree on a particular issue $[15,16]$. The key elements of this technique are: anonymity (to avoid dominance of any particular individual or group), controlled feedback (sharing distribution of group's responses with participants), iteration (to allow participants to change feedback) and statistical analysis of group response (using summary measures such as median and interquartile range). The Delphi survey is particularly useful for those situations in which there is little scientific evidence or conflicting evidence. The latter is the primary reason we chose the Delphi technique over the standard approach of analysis of patient data. It has an advantage over open committee meetings that are dominated by opinions of individuals or specific groups. However, it does not indicate "what is", rather it informs on, "what could be or should be" [16].

The purpose of the current study was to report the results of the consensus study among uveitis specialists, mainly from the Asia-Pacific region, on factors influencing the resolution of inflammation in three common clinical presentations of TBU-anterior uveitis (AU), retinal vasculitis (RV) and multifocal serpiginoid choroiditis (MSC).

\section{Materials and methods}

\section{Design and organization}

We applied a modified Delphi approach [15], to generate consensus on prognostic factors influencing the resolution of inflammation in selected clinical presentations of TBU (Fig. 1). The initial questionnaire was generated through
Fig. 1 Flowchart depicting sequence of events in Rounds 1-3 of the Delphi Survey for prognostic factors for TBassociated uveitis.

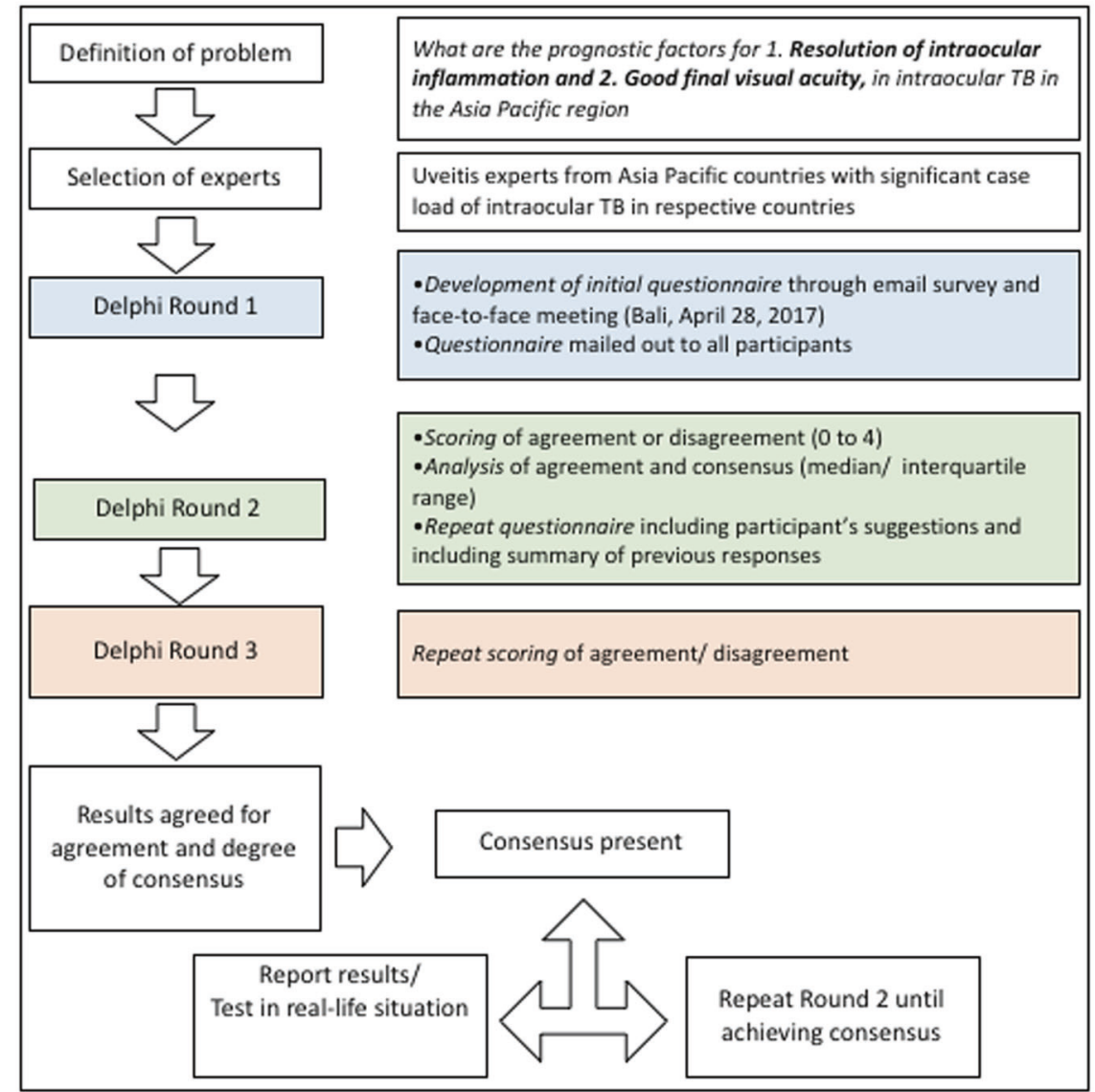


face-to-face meeting, involving all members of the expert panel. The responses were summarized after each round, and the questionnaire was truncated based on the results of the prior round (described below). Unresolved issues were discussed individually with participants, and the final list of items was approved by all.

\section{Selection of the expert panel}

The panel was selected from amongst uveitis specialists representing ten different countries, almost entirely from the Asia-Pacific region. All selected participants were trained uveitis specialists with large uveitis caseloads of TB in their respective countries. The countries included (with number of participants from each country) were: Australia (2), India (5), Indonesia (1), Japan (1), Malaysia (1), Myanmar (1), the Philippines (1), Singapore (1), Taiwan (1), Thailand (1), and USA (1). However, not all countries included in the study were high endemic for TB. For example, Australia, Japan, and USA are low-burden countries while Singapore and Taiwan are middle-burden countries. These countries were included to cover the entire spectrum of distribution of TBU in Asia-Pacific countries. Even in the low- and middle-burden countries, the participants had adequate experience in diagnosis and treatment of TBU, mainly due to their location in referral centres in their respective cities.

\section{Development of questionnaire}

The questionnaire was developed during a face-to-face meeting between the participants and other experts in the field at the Global Ocular Inflammation Workshops held at Bali, Indonesia on April 28, 2017. Prior to the meeting, we sent an email explaining the purpose of the study, along with the invitation to each of the participants. A total of 16 of the 19 invitees agreed to be part of the study. All 16 participants were present during the face-to-face meeting. During the meeting, we discussed the existing literature on TBU, and the need for identifying prognostic factors in TBU, with all participants. It was ensured that all participants agreed to the current guidelines for diagnosis and treatment of TBU $[2,3]$. All possible variables, including demographic factors, past treatment, ocular imaging, systemic evidence of TB, laboratory investigations and treatment, were presented. Our initial goal was to cover prognostic factors affecting resolution of inflammation as well as visual outcomes in TBU. However, it was determined that covering multiple outcomes and multiple clinical presentations, was beyond the scope of a single study. Therefore, we restricted the survey to factors affecting resolution of inflammation in three well-defined clinical presentations of TBU: AU, RV, and multifocal serpiginoid choroiditis. The variables selected at the end of the meeting were circulated to all participants for confirmation, and any further modifications were sought. The final questionnaire consisted of 40 putative questionnaire items that were divided into six broad categories: demographic factors, past treatment, ocular imaging, systemic evidence of TB, laboratory investigations, and treatment (Table 1). The participants were explained during the face-to-face meeting as well as in the introductory communication that the question corresponding to a given point should be read as "Does the given item represent a prognostic factor (good or poor) for the resolution of inflammation in TB-associated AU/RV/ serpiginous-like choroiditis?". This was sent out by one member of the group (RLDN), and all members were required to send back their responses to the same person. This ensured that none of the individual responses was disclosed to other members of the group.

\section{Scoring criteria}

Each item on the questionnaire was scored on a 4-point Likert scale, ranging from $0-3,(0=$ no, $1=$ minimal, $2=$ moderate, and $3=$ strong agreement with the item being a prognostic factor). The scores provided by the experts were summarized with the following two measures: median (degree of support for a given item) and interquartile range (IQR, degree of consensus among the experts).

To develop the final prognostic variable list, we ran the Delphi survey in three rounds. RLDN collated the first responses from each participant (referred as Round 1) and calculated the median and IQR for the responses on each item in the questionnaire. All 40 items from Round 1, plus their medians and IQRs, were sent back to the participants for further iteration (referred as Round 2). In addition, participants were also required to score if the given item represented a positive or a negative prognostic factor. Median and IQR were calculated again for the responses of Round 2, and the questionnaire was sent back to participants for the final round (referred as Round 3). After Round 3 , those participants who had scored $\leq 1$ for an item with median of $\geq 2$ were required to justify the reason for their disagreement. At the end of Round 3, items were considered significant to achieve consensus if they met the following criteria:

(1) Achieved a median $\geq 2$, and IQR $\leq 1$, and

(2) $\geq 75 \%$ of the respondents agreed if it is a positive or negative prognostic factor.

\section{Statistical analysis}

We analyzed the scoring data provided by experts in each round descriptively. The scoring data were displayed as median and IQR. To compare individual responses between 
Table 1 Initial set of questionnaire items $(n=40)$, that were scored in Rounds 1 and 2 of the study.

\begin{tabular}{|c|c|c|c|}
\hline Categories & Items & & \\
\hline \multirow[t]{19}{*}{ Demographic factors $(n=14)$} & TB-endemic region & & \\
\hline & Age & & \\
\hline & & $>50$ years & \\
\hline & & $<18$ years & \\
\hline & Gender & & \\
\hline & & Male & \\
\hline & & Female & \\
\hline & Duration of TB-associated uveitis & & \\
\hline & & $\geq 3$ months & \\
\hline & Previous treatment & & \\
\hline & & Corticosteroids & \\
\hline & & Immunosuppressives & \\
\hline & & Anti-TB therapy & \\
\hline & & & $<9$ months \\
\hline & & & $<6$ months \\
\hline & Past systemic TB & & \\
\hline & Co-existent systemic disease & & \\
\hline & & Diabetes & \\
\hline & & HIV & \\
\hline \multirow[t]{19}{*}{ Ocular Imaging $(n=12)$} & FFA & & \\
\hline & & Capillary non-perfusion & \\
\hline & & Cystoid macular edema & \\
\hline & Autofluorescence (after initiation of anti-TB therapy) & & \\
\hline & & Diffuse hyperautofluorescence beyond 3 months & \\
\hline & & Mottled hyperautofluorescence beyond 6 months & \\
\hline & ОСТ & & \\
\hline & & Outer retinal hyper-reflectivity & \\
\hline & & Cystoid macular edema & \\
\hline & OCT-angiography & & \\
\hline & & Flow void areas & \\
\hline & & Tangled vascular network & \\
\hline & EDI-OCT & & \\
\hline & & Diffuse choroidal thickening & \\
\hline & & Well-defined homogenous reflectivity (granuloma) & \\
\hline & ICG & & \\
\hline & & Persistent hypocyanescence & \\
\hline & Widefield imaging (photography/angiography) & & \\
\hline & & Peripheral (beyond equator) active lesion & \\
\hline \multirow[t]{11}{*}{ Systemic evidence of TB $(n=9)$} & Radiological evidence & & \\
\hline & & Pulmonary TB-active & \\
\hline & & Pulmonary TB—healed & \\
\hline & & Mediastinal TB & \\
\hline & & Extrapulmonary TB & \\
\hline & Microbiological evidence (smear/culture/sensitivity) & & \\
\hline & & Pulmonary & \\
\hline & & Extrapulmonary & \\
\hline & & Multidrug resistant & \\
\hline & High TST value ( $20 \mathrm{~mm}$, necrotic) & & \\
\hline & High IGRA value ( $>8 \mathrm{IU} / \mathrm{mL}$, based on Gineys et al., AJO, 2011) & & \\
\hline \multicolumn{4}{|l|}{ Laboratory investigations $(n=1)$} \\
\hline & M. tuberculosis PCR & & \\
\hline & & High value on quantitative PCR & \\
\hline \multicolumn{4}{|l|}{ Current treatment $(n=5)$} \\
\hline & Anti-TB therapy (ATT) & & \\
\hline & & Duration $\geq 9$ monnths & \\
\hline & & Ethambutol during maintenance phase & \\
\hline & Corticosteroids_-systemic ( $10 \mathrm{mg} / \mathrm{day}, 3$ months after of initiation of ATT) & & \\
\hline & Corticosteroids-local ( 3 months after of initiation of ATT) & & \\
\hline & Immunosuppressives & & \\
\hline
\end{tabular}

FFA Fundus fluorescein angiography, OCT Optical Coherence Angiography, EDI-OCT Enhanced depth Imaging-OCT, ICG Indocyanine green angiography, TST Tuberculin Skin Test, IGRA Interferon Gamma Release Assay, PCR Polymerase Chain Reaction 
Table 2 Progression in number of items with consensus (IQR $\leq$ 1) and significant scores (median $\geq 2$ ) in each clinical category, from Round 1-3.

\begin{tabular}{|c|c|c|c|c|c|c|}
\hline & \multicolumn{3}{|c|}{$\begin{array}{l}\text { Number of items with consensus } \\
\text { (IQR } \leq 1 \text {, irrespective of } \\
\text { median score) }\end{array}$} & \multicolumn{3}{|c|}{$\begin{array}{l}\text { Number of items with significant } \\
\text { scores }(\text { median } \geq 2)\end{array}$} \\
\hline & Round 1 & Round 2 & Round 3 & Round 1 & Round 2 & Round 3 \\
\hline Anterior uveitis & 3 & 9 & 18 & 11 & 17 & 17 \\
\hline Retinal vasculitis & 3 & 21 & 24 & 28 & 31 & 31 \\
\hline Multifocal serpigenoid choroiditis & 11 & 26 & 27 & 32 & 34 & 34 \\
\hline
\end{tabular}

participants from endemic and nonendemic countries, we compared the scores by Fisher's exact test. We used SPSS 22 (IBM Corp, Armonk, NY, US) software to analyze the data.

\section{Results}

Each of the 16 participants completed all three rounds of scoring.

Round 1: The number of items with significant consensus (IQR $\leq 1)$, and with significant scores (median $\geq 2$ ) at the end of each round, is summarized in Table 2. The number of items that scored median $\geq 2$ after Round 1 was similar to the numbers that scored after Rounds 2 and 3 (except for AU). However, a relatively small number of items achieved consensus on scoring $(\mathrm{IQR} \leq 1)$ between participants after Round 1: of the total of 40, only three achieved consensus for both $\mathrm{AU}$ and $\mathrm{RV}$, and 11 achieved consensuses for MSC.

Round 2: All 40 items, with their median and IQR values, were presented to the participants for re-scoring. After Round 2, the number of items with median $\geq 2$ increased significantly for AU, and marginally for RV and MSC categories (Table 2). In contrast, the number of items achieving significant consensus $(\mathrm{IQR} \leq 1)$ increased substantially in all three categories.

Round 3: In this round, only items with median $\geq 2$ were presented to the participants. The participants were required to review their scoring for all items, specifically those with IQR $\geq 1$ (significant disagreement, despite median $\geq 2$ ). As in Round 2, the degree of consensus further improved in all three categories, as compared with the previous round, although the number of items with significant median scores $(\geq 2)$ remained the same.

The number of items that had both median $\geq 2$ and IQR $\leq 1$, after Round 3 were 15, 21, and 20 for AU, RV, and MSC, respectively (Table 3). However, in only 4, 9, and 8 items in each of these respective clinical phenotypes, did the participants agreed that these were positive or negative prognostic factors. These items formed the final list of prognostic factors for the resolution of inflammation in intraocular TB and are highlighted in red or green respectively to represent negative or positive prognostic factors, respectively. They included: delay in diagnosis ( $\geq 3$ months), previous corticosteroid or immunosuppressive therapy, specific imaging characteristics and co-existing HIV infection for $\mathrm{RV}$ and MSC, microbiologically proven multidrug resistant TB, and current anti-TB therapy $\geq 9$ months. All except the last item were considered to be negative prognostic factors for resolution of inflammation.

\section{Comparison between endemic and nonendemic countries}

Comparison of responses with each item in the list by participants from endemic $(n=9)$ and nonendemic $(n=7)$ countries did not reveal significant differences for any item between the two groups (Supplementary Table 1).

\section{Discussion}

\section{Key findings}

Expert consensus indicated that the resolution of inflammation in intraocular TB may be affected by a wide range of factors, such as delayed diagnosis of uveitis, past corticosteroid or immunosuppressive therapy, specific imaging features in different clinical presentations, co-infection with HIV, multidrug resistant TB, and the duration of ATT used in the treatment of uveitis. In addition, several factors that support an etiological diagnosis of intraocular TB, such as co-existing pulmonary, mediastinal or other extrapulmonary $\mathrm{TB}$, and high values on quantitative PCR for TB, also achieved consensus on their significance, but there was a lack of consensus on whether these were positive or negative prognostic factors. Items that were excluded during the survey included age of patient, pre-existing diabetes, and high values for tuberculin skin test (TST) or interferon gamma release assays (IGRA).

\section{Comparison with existing literature}

Delays in the diagnosis of intraocular $\mathrm{TB}$ and in the initiation of anti-TB therapy are largely responsible for the visual morbidity associated with this condition [17]. 
Table 3 Final list of prognostic factors, that met the selection criteria, at the end of Round 3 (Achieved a median $\geq 2$, and IQR $\leq 1$; and $\geq 75 \%$ of the respondents agreed to either a positive or negative prognostic factor).

\begin{tabular}{|c|c|c|c|c|}
\hline & Demographic factors & Anterior uveitis & $\begin{array}{l}\text { Retinal } \\
\text { vasculitis }\end{array}$ & $\begin{array}{l}\text { Multifocal serpigenoid } \\
\text { choroiditis }\end{array}$ \\
\hline 1 & TB-endemic region & $2 ; 2-3(69)$ & $3 ; 2-3(46.7)$ & $3 ; 3-3(46.7)$ \\
\hline \multirow[t]{2}{*}{2} & Duration of uveitis $\geq 3$ months & $2 ; 2-3(86.7)$ & $3 ; 2-3(87.5)$ & $3 ; 3-3(80)$ \\
\hline & Previous treatment & & & \\
\hline 3 & Corticosteroids & $2 ; 2-2$ (76.9) & $3 ; 2-3(62.5)$ & $3 ; 2-3$ (73.3) \\
\hline 4 & Immunosuppressive & $2 ; 2-2(84.6)$ & $2 ; 2-3(75)$ & $3 ; 2-3(80)$ \\
\hline 5 & ATT $<6$ months & $2 ; 2-2.25(64.2)$ & $2 ; 2-3(73.3)$ & $3 ; 2-3$ \\
\hline 6 & Past systemic TB & $2 ; 2-2.25(58.3)$ & $2 ; 2-2(73.3)$ & $2 ; 2-3$ \\
\hline 7 & Co-existent HIV & $2.5 ; 2-3(71.4)$ & $3 ; 2.75-3(78.6)$ & $3 ; 2-3(78.6)$ \\
\hline \multirow[t]{2}{*}{8} & Ocular imaging & & & \\
\hline & FFA: Capillary nonperfusion & --- & $3 ; 2-3(81.3)$ & --- \\
\hline 9 & FFA: Cystoid macular oedema & & $2 ; 2-3(75)$ & \\
\hline 10 & $\begin{array}{l}\text { AF: Persistent hyper-AF beyond } 6 \text { months of } \\
\text { starting ATT }\end{array}$ & --- & --- & $2.5 ; 2-3(80)$ \\
\hline 11 & OCT-Outer retinal hyper-reflectivity & --- & --- & $3 ; 2-3(78.6)$ \\
\hline 12 & OCT - Cystoid macular oedema & --- & $2 ; 2-3(75)$ & $2 ; 2-3(73.3)$ \\
\hline 13 & Widefield imaging: active lesions beyond equator & --- & $3 ; 2-3(76.9)$ & $2 ; 2-3(75)$ \\
\hline \multirow[t]{2}{*}{14} & Systemic evidence & & & \\
\hline & Pulmonary TB -active & $2 ; 2-3(64.2)$ & $3 ; 2-3(57.1)$ & $3 ; 2-3(57.1)$ \\
\hline 15 & Mediastinal TB & --- & $3 ; 2-3(50)$ & $3,3-3(50)$ \\
\hline 16 & Extrapulmonary TB & $2.5 ; 2-3(53.8)$ & $2.5 ; 2-3(60)$ & $3 ; 2-3(57.1)$ \\
\hline \multirow[t]{2}{*}{17} & $\begin{array}{l}\text { Microbiological evidence (smear/ culture/ } \\
\text { sensitivity) }\end{array}$ & & & \\
\hline & Pulmonary & $2.5 ; 2-3(50)$ & --- & --- \\
\hline 18 & Extrapulmonary & $2 ; 2-3(64.2)$ & $2 ; 2-3(64.2)$ & $2 ; 2-3(57.1)$ \\
\hline 19 & Multi-drug resistant & $3 ; 3-3(100)$ & $3 ; 3-3(100)$ & $3 ; 3-3(100)$ \\
\hline \multirow[t]{2}{*}{20} & Laboratory investigations & & & \\
\hline & Quantitative PCR: high value & --- & $2 ; 2-3(61.5)$ & $2 ; 2-3(61.5)$ \\
\hline \multirow[t]{2}{*}{21} & Current treatment & & & \\
\hline & ATT $\geq 9$ months & $2 ; 2-3(73.3)$ & $2 ; 2-3(80)$ & $2 ; 2-3(78.6)$ \\
\hline 22 & $\begin{array}{l}\text { Oral corticosteroids }>10 \mathrm{mg} / \text { day } 3 \text { months after } \\
\text { initiation of ATT }\end{array}$ & --- & $2 ; 2-2(56.3)$ & $2 ; 2-3(46.7)$ \\
\hline 23 & $\begin{array}{l}\text { Local corticosteroids } 3 \text { months after initiation of } \\
\text { ATT }\end{array}$ & $2 ; 1.75-2(57.1)$ & $2 ; 1.25-2(62.5)$ & --- \\
\hline 24 & Immunosuppressive therapy & --- & $3 ; 2-3(62.5)$ & $3 ; 2.75-3(64.3)$ \\
\hline
\end{tabular}

The numbers represent median, interquartile range and \% of respondents agreeing to the item being a positive or negative prognostic factor (in brackets). The positive prognostic factors are shaded green and the negative factors orange. The remainder which did not meet our significance criteria, have not been highlighted

The diagnosis of intraocular TB is based on clinical signs and ancillary tests in majority cases-all of which only provide indirect evidence of mycobacterial infection in the eye [2]. Not surprisingly, factors suggestive of an etiological diagnosis of intraocular TB were judged to have a strong influence on prognosis, though there was a lack of clarity on the direction of the effect. Interestingly, all except TB PCR from ocular fluids, are related to evidence of systemic, not ocular TB infection, which highlights the diagnostic constraints in this condition. However, TST and IGRA, the two most commonly used tests for screening TB in uveitis patients, were not scored sufficiently to meet the 
prognostic criteria. This possibly reflects the limited utility of these tests to rule in the disease, in the absence of other corroborative evidence (low pre-test probability) [18, 19].

Another set of prognostic factors included various imaging modalities used in intraocular TB. Imaging modalities not only help identifying the disease pattern and extent in intraocular TB, they also demonstrate structural changes in the affected tissues, as the disease progresses or responds to treatment [20, 21]. In RV, these changes included capillary non-perfusion on FFA, cystoid macular edema on FFA, or OCT. In case of MSC, persistent fundus autofluorescence (>6 months after starting ATT), outer retinal hyperreflectivity on OCT, and lesions beyond equator on widefield imaging, were scored significantly. Based on face-toface discussion, we had included only selected imaging items in the initial questionnaire amongst the multiple features that have been characterized for intraocular TB.

Our survey also reaffirmed the role of ATT in the resolution of inflammation in intraocular TB. This has been described in a number of retrospective studies including recent multicentred trials, though the quality of evidence in this field is compromised by the lack of appropriate controls and grading of inflammation [8,9]. The participants also identified need for prolonged immunosuppression as a prognostic factor for the resolution of inflammation. This corroborates with an earlier report that patients taking systemic immunosuppressive therapy had higher odds of treatment failure following ATT [22], and probably reflects the uncertainty in diagnosing such cases, and therefore poor response to standard therapy. Alternatively, it could imply that the inflammatory response is disproportionately high in such cases, and that prolonged anti-inflammatory therapy is needed.

\section{Strengths and limitations of the study}

The main strength of this study is the enumeration of prognostic factors for a disease, where such data are not available in current literature and would be difficult to derive directly from patient data. While recent multi-centre studies have attempted to evaluate prognostic factors for intraocular TB based on patient data, they have been limited by the lack of standardization, control groups and evaluation of individual phenotypes [8,9]. The modified Delphi technique used in this survey largely eliminated any bias or any dominating influence of individuals as typically occurs in face-to-face meetings. We included uveitis experts from countries across Asia-Pacific region, with varying TB endemicities. The Delphi technique has been widely used in medicine, including a number of ophthalmic conditions such as uveitis (Standardization of Uveitis Nomenclature classification), ocular surface disease, autoimmune retinopathy, and thyroid eye disease [23-26]. All these conditions share the common feature of having variable clinical presentations, and the Delphi technique has typically been used for listing diagnostic or grading criteria based on expert consensus. However, to date, this technique has not been utilized for evaluating prognostic factors for any ocular condition.

A major challenge was that we did not achieve consensus on several items being positive or negative prognostic factors, despite them achieving consensus and significance on scoring. Most of the items that remained ambiguous about the direction of effect, were those related to radiological or microbiological evidence of systemic TB. This is understandable: on one hand, such evidence increases the likelihood of etiological diagnosis of TB, and therefore timely initiation of appropriate anti-TB therapy; and the other hand, the presence of systemic TB may represent a higher mycobacterial load in the body, and therefore worse prognosis. This is a unique challenge while applying the Delphi technique to prognostic factor estimation, as it requires two simultaneous scores (significance of score on Likert scale, and positive or negative effect on prognosis) for the same variable. This may explain why Delphi surveys for prognostic factors are uncommon. Another major limitation is that, a Delphi survey estimates only 'what could/should be', not 'what is'. The results of such surveys will have to be validated with prospectively collected patient data. Other major limitations that are generic to all Delphi surveys are the possibility of respondent fatigue during successive rounds of iteration and the requirement for longer study durations than telephonic interviews or face-to-face questionnaires. Future surveys should ensure sufficient spacing between each round of survey and persistent motivation by the lead investigators during the course of the survey.

\section{Implications for clinical practice and future research}

Our study provides an agreed list of prognostic factors that experts from TB-endemic and nonendemic countries consider most significant for resolution of inflammation in intraocular TB. It emphasizes that early etiological diagnosis of TB and initiation of ATT are most crucial for resolution of inflammation. It also highlights the role of specific imaging modalities in prognosticating different clinical presentations of intraocular TB. In the future, individual items identified in this survey will be validated through prospective data collected at various clinics across Asia-Pacific region. Future research directed at point-of-care diagnostics from ocular fluids may help in achieving greater etiological accuracy and therefore better prognosis. Advances in imaging modalities should also improve prediction of course of disease and response to therapy. 


\section{Summary}

\section{What was known before}

- Tuberculosis (TB) is associated with a wide variety of intraocular inflammation. Diagnosis of TB-associated uveitis (TBU) is challenging anti-TB therapy (ATT) that leads to resolution of inflammation and prevention of recurrences in TBU.

\section{What this study adds}

- Duration of disease, previous corticosteroid/immunosuppressive therapy, imaging features, co-existent HIV, and multidrug resistant TB are poor prognostic factors in TBU Duration of ATT $\geq 9$ months is a good prognostic factor for resolution of inflammation in TBO. These factors need validation in prospective clinical trials.

Author contributions SB: Conception of study; development of questionnaire; participation in survey; data analysis; manuscript writing and review; moderating face-to-face meeting of International Ocular TB Study Group. RLDN: Conception of study; development of questionnaire; dissemination of questionnaire and collection of data; data analysis; manuscript writing and review. NR: Development of questionnaire; participation in survey; data analysis; manuscript writing and review. XJ: Statistical analysis; manuscript review. AF: Statistical analysis; manuscript review.

Members of the International Ocular TB Study Group Peter J. McCluskey ${ }^{6}$, Justine R. Smith ${ }^{7}$, Soumyava Basu ${ }^{8}$, Jyotirmay Biswas ${ }^{9}$, Padmamalini Mahendradas ${ }^{10}$, Salil Mehta ${ }^{11}$, Kalpana Babu Murthy ${ }^{12}$, Lukman Edwar ${ }^{13}$, Rina La Distia Nora ${ }^{14}$, Kazuichi Murayama ${ }^{15}$, Shelina Oli Mohamed ${ }^{16}$, May Z. A. Win ${ }^{17}$, Jessica Marie Abaño ${ }^{18}$, Soon-Phaik $\mathrm{Chee}^{19}$, Shwu Jiuan Sheu ${ }^{20}$, Somsiri Sukavatcharin ${ }^{21}$, Narsing A. Rao ${ }^{22}$.

${ }^{6}$ Save Sight Institute, The University of Sydney, Discipline of Clinical Ophthalmology and Eye Health, Sydney, Australia; ${ }^{7}$ Flinders University College of Medicine and Public Health, Adelaide, Australia; ${ }^{8}$ Retina and Uveitis services, L V Prasad Eye Institute, Bhubaneswar, India; ${ }^{9}$ Uveitis \& Ocular Pathology, Sankara Nethralaya, Chennai, India; ${ }^{10}$ Uveitis and Ocular immunology, Narayana Nethralaya, Bangalore, India; ${ }^{11}$ Department of Ophthalmology, Lilavati Hospital, Mumbai, India; ${ }^{12}$ Prabha Eye Clinic \& Research Centre \& Vittala International Institute of Ophthalmology, Bengaluru, India; ${ }^{13}$ Department of Ophthalmology, Faculty of Medicine, Universitas Indonesia, Cipto Mangunkusumo Hospital, Jakarta, Indonesia; ${ }^{14}$ Department of Ophthalmology, Universita Indonesia \& Cipto Mangunkusumo Hospital Kirana, Jakarta, Indonesia; ${ }^{15}$ Department of Innovative Visual Science, Graduate School of Medicine, Osaka University, Osaka, Japan; ${ }^{16}$ Department of Ophthalmology, Hospital Shah Alam, Selangor, Malaysia; ${ }^{17}$ Ophthalmology Department, University of Medicine 1, Yangon, Myanmar; ${ }^{18}$ Ocular Inflammation and Immunology Service, St. Luke's Medical Center -Bonifacio Global City, Taguig, Manila, Philippines; ${ }^{19}$ Department of Ophthalmology, Yong Loo Lin School of Medicine, National University of Singapore, Singapore Eye Research Institute, Singapore, Singapore; ${ }^{20}$ Department of Ophthalmology, Kaohsiung Medical University, Chung Ho Memorial Hospital, Kaohsiung, Taiwan; ${ }^{21}$ Uveitis and Ocular immunology, Faculty of Medicine, Ramathibodi Hospital, Mahidol University, Bangkok, Thailand; ${ }^{22}$ USC-Roski Eye Institute, Department of Ophthalmology, Keck School of Medicine, University of Southern California, Los Angeles, CA, USA

\section{Compliance with ethical standards}

Conflict of interest The authors declare that they have no conflict of interest.

Publisher's note Springer Nature remains neutral with regard to jurisdictional claims in published maps and institutional affiliations.

\section{References}

1. Majumdar SS, Marais BJ, Denholm JT, Britton WJ. Drug-resistant tuberculosis: collaborative regional leadership required. Med J Aust. 2014;200:241-2.

2. Gupta A, Sharma A, Bansal R, Sharma K. Classification of intraocular tuberculosis. Ocul Immunol Inflamm. 2015;23:7-13.

3. Gupta A, Bansal R, Gupta V, Sharma A, Bambery P. Ocular signs predictive of tubercular uveitis. Am J Ophthalmol. 2010;149: 562-70.

4. Vasconcelos-Santos DV, Rao PK, Davies JB, Sohn EH, Rao NA. Clinical features of tuberculous serpiginous-like choroiditis in contrast to classic serpiginous choroiditis. Arch Ophthalmol. 2010;128:853-8.

5. Nora RL, van Velthoven ME, Ninette H, Misotten T, Bakker M, van Hagen MP, et al. Clinical manifestations of patients with intraocular inflammation and positive QuantiFERON-TB gold intube test in a country nonendemic for tuberculosis. Am J Ophthalmol. 2014;157:754-61.

6. Bansal R, Gupta A, Gupta V, Dogra MR, Bambery P, Arora SK. Role of anti-tubercular therapy in uveitis with latent/manifest tuberculosis. Am J Ophthalmol. 2008;146:772-9.

7. Kee AR, Gonzalez-Lopez JJ, Al-Hity A, Gupta B, Lee CS, Gunasekeran DV, et al. Anti-tubercular therapy for intraocular tuberculosis: a systematic review and meta-analysis. Surv Ophthalmol. 2016;61:628-53.

8. Agrawal R, Gunasekeran DV, Grant R, Agarwal A, Kon OM, Nguyen QD, et al. Clinical features and outcomes of patients with tubercular uveitis treated with antitubercular therapy in the Collaborative Ocular Tuberculosis Study (COTS)-1. JAMA Ophthalmol. 2017;135:1318-27.

9. Tomkins-Netzer O, Leong BCS, Zhang XZ, Lightman S, McCluskey PJ. for the Sydney-London Latent Ocular TB Study Group. Am J Ophthalmol. 2018;190:164-70.

10. Bajema KL, Pakzad-Vaezi K, Hawn T, Pepple KL. Tuberculous uveitis: association between anti-tuberculous therapy and clinical response in a non-endemic country. J Ophthalmic Inflamm Infect. 2017;7:19.

11. Krassas N, Wells J, Bell C, Woodhead M, Jones N. Presumed tuberculosis-associated uveitis: rising incidence and widening criteria for diagnosis in a non-endemic area. Eye. 2018;32:87-92.

12. Basu S, Nayak S, Padhi TR, Das T. Progressive ocular inflammation following anti-tubercular therapy for presumed ocular tuberculosis in a high-endemic setting. Eye. 2013;27:657-62.

13. Tagirasa R, Parmar S, Barik MR, Devadas S, Basu S. Autoreactive $T$ cells in immunopathogenesis of TB-associated uveitis. Invest Ophthalmol Vis Sci. 2017;58:5682-91.

14. Basu S, Monira S, Modi RR, Choudhury N, Mohan N, Padhi TR, et al. Degree, duration, and causes of visual impairment in eyes affected with ocular tuberculosis. J Ophthalmic Inflamm Infect. 2014;4(Feb):3 
15. Jones J, Hunter D. Consensus methods for medical and health services research. BMJ. 1995;311:376-80.

16. Hsu CC, Sandford BA. The Delphi technique: making sense of consensus. Pr Assess Res Eval. 2007;12:1-8.

17. Patel SS, Saraiya NV, Tessler HH, Goldstein DA. Mycobacterial ocular inflammation: delay in diagnosis and other factors impacting morbidity. JAMA Ophthalmol. 2013;131:752-8.

18. Rosenbaum JT, Wernick R. The utility of routine screening of patients with uveitis for systemic lupus erythematosus or tuberculosis: a Bayesian analysis. Arch Ophthalmol. 1990;108:1291-3.

19. Agrawal R, Grant R, Gupta B, Gunasekeran DV, Gonzalez-Lopez JJ, Addison PKF, et al. What does IGRA testing add to the diagnosis of ocular tuberculosis? A Bayesian latent class analysis. BMC Ophthalmol. 2017;17:245.

20. Agarwal A, Mahajan S, Khairallah M, Mahendradas P, Gupta A, Gupta V. Multimodal imaging in ocular tuberculosis. Ocul Immunol Inflamm. 2017;25:134-45.

21. Bansal R, Basu S, Gupta A, Rao N, Invernizzi A, Kramer M. Imaging in tuberculosis-associated uveitis. Indian J Ophthalmol. 2017;65:264-70.
22. Agrawal R, Gupta B, Gonzalez-Lopez JJ, Rahman F, Phatak S, Triantafyllopoulou I, et al. The role of anti-tubercular therapy in patients with presumed ocular tuberculosis. Ocul Immunol Inflamm. 2015;23:40-6.

23. Standardization of Uveitis Nomenclature (SUN) Working Group. Standardization of uveitis nomenclature for reporting clinical data. Results of the First International Workshop. Am J Ophthalmol. 2005;140:509-16.

24. Behrens A, Doyle JJ, Stern L, Chuck RS, McDonnell PJ, Azar DT, et al. Dysfunctional tear syndrome: a Delphi approach to treatment recommendations. Cornea. 2006;25:900-7.

25. Douglas RS, Tsirbas A, Gordon M, Lee D, Khadavi N, Garneau $\mathrm{HC}$, et al. Development of criteria for evaluating clinical response in thyroid eye disease using a modified Delphi technique. Arch Ophthalmol. 2009;127:1155-60.

26. Fox AR, Gordon LK, Heckenlively JR, Davis JL, Goldstein DA, Lowder CY, et al. Consensus on the diagnosis and management of nonparaneoplastic autoimmune retinopathy using a modified Delphi approach. Am J Ophthalmol. 2016;168: 183-90. 